\title{
Contributed Papers for JD10: Abstracts
}

\author{
A Detailed Study of the Massive Lensing Cluster Cl 0024+16 \\ R. S. Ellis (Caltech), T. Treu (Caltech), J-P. Kneib (OMP)
}

\begin{abstract}
We present a comprehensive study of galaxies and dark matter to the turn-around radius $(5 \mathrm{Mpc})$ in the lensing cluster Cl $0024+16(z=0.40)$. We directly study the transformation of field galaxies as they fall into the potential determined quantitatively from weak lensing signals. Our analysis exploits a mosaic of 38 WFPC2 images and ground-based imaging and spectroscopy using CFHT, Keck and Palomar. Correlations between morphology, star formation rate and environmental location are interpreted using known infall time scales. The dark matter distribution, revealed for the first time over such a wide dynamical range in density, is compared directly with the numerical simulations and with that observed for the stellar light. In-depth studies of more clusters, sampling a wide range in environmental density, will yield valuable insights into the origin of the morphology-density relation observed today.
\end{abstract}

\section{Galaxy Groups: New Insights from Chandra and XMM}

J. M. Vrtilek (CFA), E. J. O'Sullivan (CFA), L. P. David (CFA), T. J. Ponman (Birmingham), W. R. Ponman (CFA), C. Jones-Forman (CFA), D. E. Harris (CFA)

\begin{abstract}
Groups and poor clusters are the locus of most galaxies in the present-day Universe and the building blocks from which clusters form. They accordingly occupy a significant place in the continuum of structure between isolated galaxies and rich clusters. Owing to the lower temperature of their intracluster gas, X-ray emission from groups produces strong lines from a broader range of elements than do hotter clusters. Here, we employ Chandra and XMM images of several X-ray bright groups (including HCG 62, NGC 741, and MKW 4) to examine issues of current interest in the study of both groups and clusters: the distribution of heavy elements, the presence and nature of X-ray cavities and their relation to radio observations, the presence of cooling cores, and X-ray signatures of recent galaxy interactions. This work has been supported in part by NASA grants GO0-1037X, GO1-2138X, and GO2-3186X.
\end{abstract}

\section{Cluster Properties via Sunyaev-Zel'dovich Effect \\ Asantha Cooray (Caltech) California Instiute of Technology}

\begin{abstract}
We will discuss the role played by the Sunyaev-Zel'dovich (SZ) effect in understanding the physical properties of the intra-cluster medium. While the SZ effect has been considered widely for its cosmological purposes, when combined with multi-wavelength observations, the SZ effect data can also be used to understand the nature and evolution of the ICM including its thermal structure and the presence of non-thermal plasma. We also discuss future opportunities on this aspect involving observations from the planned South Pole Telescope, Planck mission, and various other attempts to image the SZ effect in galaxy clusters using wide-field bolometer arrays and other techniques. We will also explore the connection between gas in clusters and the general intergalactic
\end{abstract}


medium and how one can use detailed wide-field SZ maps, beyond those towards individual clusters, to study such possibilities.

\title{
ACS Observations of Three Rich Galaxy Clusters at High Redshift
} J. P. Blakeslee (JHU), M. Postman (STScI), P. Rosati (ESO), H. Ford (JHU), G. Illingworth (UCSC), M. Franx (Leiden), C. Gronwall (PSU), B. Holden (UCSC)

\begin{abstract}
Our Advanced Camera for Surveys (ACS) cluster survey aims to establish new and detailed constraints on the formation and evolution of clusters and their member galaxies. We have used the ACS Wide Field Camera to obtain multi-band imaging of several X-ray selected clusters near $z \sim 1$. These clusters include MS1054-0321 and CL0152-1357, both at $z=0.83$, and CL1252-2927 at $z=1.24$. Each was observed in a mosaic of 4 partially overlapping pointings, with the central 1 arc min imaged to the greatest depth. CL1252-2927 is one of the most distant clusters known, yet it appears to be a relaxed, centrally concentrated system with a well-defined red sequence of evolved galaxies in the color-magnitude (CM) diagram. Monte Carlo simulations with the latest population models suggest an age scatter of about $30 \%$ for the ellipticals, with a mean age of at least $2.5 \mathrm{Gyr}$, for a formation epoch $z>2.7$. The slope and scatter in the elliptical CM relation show little or no evidence for evolution out to $z=1.2$. However, the clusters at $z=0.83$ show signs of ongoing dynamical evolution, including irregular structure and significant merging, although they also show prominent red sequences.
\end{abstract}

\section{The Role of Dwarf Galaxies in Galaxy Cluster Evolution Christopher Conselice (Caltech)}

\begin{abstract}
Although they are the faintest galaxies in clusters, dwarf galaxies, especially dwarf ellipticals, are a key component for understanding the evolution of cluster galaxies, as well as clusters themselves. These dwarfs dominate clusters in number, and potentially contain a significant fraction of the cluster's dark matter. They are also potentially the remnants of larger infalling Butcher-Oemler galaxies stripped of their mass through dynamical cluster processes. We will present observational results that show that dwarfs in nearby clusters could be the remnants of much of early galaxy formation in clusters and discuss how these objects can account for many observed properties of clusters such as intergalactic light.
\end{abstract}

\section{Ultra-compact Dwarf Galaxies: New Constituents of Clusters}

M. J. Drinkwater (U Queensland), M. D. Gregg (UCD), M. Hilker (Bonn), W. J. Couch (UNSW), H. C. Ferguson (STScI), B. Jones (Nottingham), S. Phillipps (Bristol)

\footnotetext{
Abstract. In the course of our all-object Fornax Cluster Spectroscopic Survey, using the Anglo-Australian Telescope's $2 \mathrm{dF}$ spectrograph, we discovered a new population of physically small ultra-compact dwarf (UCD) galaxies. Unresolved from the ground, these objects have been overlooked by previous galaxy surveys although clearly visible on 30 -year-old sky survey plates. We present high-resolution imaging (HST) and spectroscopy (VLT and Keck) of the Fornax UCDs. They are qualitatively different from both globular star clusters and all
} 
known galaxy types: we have discovered an entirely new class of galaxy. The data are consistent with our hypothesis that UCDs are the remnant nuclei of nucleated dwarf galaxies which have been tidally stripped by the central galaxy of the cluster. We suggest that part of the huge globular clusters populations around central cluster galaxies are really UCDs and not globular clusters at all. Based on our UCD formation hypothesis, we predicted that a similar population would exist in the Virgo Cluster. In just 3 hours of $2 \mathrm{dF}$ service time we measured redshifts of 900 objects towards the Virgo Cluster and identified 9 UCDs. UCDs appear to be a common product of galaxy evolution in the cluster environment.

\title{
The Evolution of Active Galaxies in Clusters Philip Best (IfA Edinburgh)
}

\begin{abstract}
Recent deep studies at radio, sub-mm and X-ray wavelengths of both low and high $(z \sim 1)$ redshift clusters are reviewed, to investigate the active galaxy content of these clusters. These results show that clusters, especially at intermediate and high redshifts, contain a significant population of powerful active galaxies, which would not be identified as such by either imaging or spectroscopy at optical wavelengths. Studies of this active galaxy population are on-going, and offer the opportunity to shed light on the triggering, fueling and suppression of active and star-forming galaxies, as well as on the evolutionary status of the high redshift clusters themselves.
\end{abstract}

\section{Chandra X-ray Observations of 5 Radio Galaxies with $z=2-2.6$}

D. E. Harris (SAO), R. Overzier (Leiden), C. L. Carilli (NRAO), J. Kurk (Leiden), G. K. Miley (Leiden), L. Pentericci (MPIA Heidelberg), H. Rottgering (Leiden), and W. van Breugel (LLNL)

\begin{abstract}
We report on Chandra observations of $20 \mathrm{ks}$ each on the high redshift radio galaxies $0156-252,0406-244,0828+193,2036-254$, and 2048-272. These data were obtained to evaluate the amount of hot gas around the radio galaxies (none was found, $M<$ few $\times 10^{12} \mathrm{M}_{\odot}$ ); to determine if there is an over-density of AGN in the fields surrounding the radio galaxies (preliminary results are negative); and to ascertain if some of the X-ray emission could be non-thermal (we detected emission consistent with the IC/CMB process for hotspots/lobes in 4 of the 5 targets). In 4 cases the nucleus of the host galaxy is detected, and for 3 targets there is off-nuclear emission aligned with the radio axes. One extended X-ray source was detected adjacent to a radio galaxy. If it were at the same redshift, it would be of order $100 \mathrm{kpc}$ in diameter with $L_{\mathrm{X}}=10^{44} \mathrm{erg} \mathrm{s}^{-1}$. The work at SAO was partially supported by NASA grant GO2-3139B and contract NAS8-39073.
\end{abstract}

\section{Cooling Cores and Radio Mini-Halos}

M. Gitti (Innsbruck; IRA-CNR Bologna), G. Brunetti (IRA-CNR Bologna), G. Setti (Bologna), L. Feretti (IRA-CNR Bologna)

Abstract. We have recently developed a model for the origin of radio minihalos, observed in some cooling flow clusters, as due to synchrotron emission from relativistic electrons re-accelerated by MHD turbulence. The MHD turbulence, naturally present in the ICM, is assumed to be frozen into the compressed 
thermal ICM and thus amplified in the cooling region. Here we present the application of this model to the mini-halo candidates observed in the Perseus cluster and in A2626. A very good agreement between the model and observations is found for the three observables: surface brightness profile, integrated spectrum and radial spectral steepening. Our results showed that a relic population of relativistic electrons can be efficiently re-accelerated by MHD turbulence, with the necessary power being only a small fraction of the maximum power that can be extracted by the cooling flow. Observationally, we notice that the strongest radio mini-halos are found in association with the most powerful cooling flows, and that cooling flow powers are orders of magnitude larger than the integrated radio power.

\title{
Abell 754: Low Frequency Observations of a Merging Cluster T. E. Clarke (U Virginia), N. Kassim (NRL), T. Ensslin (MPIA Garching), D. Neumann (CEA Saclay)
}

\begin{abstract}
In the hierarchical model of structure formation, discrete sources form from the collapse of initial density enhancements and subsequently grow through gravitational effects. Clusters of galaxies are thought to form at the intersection of large scale filaments and are expected to undergo a number of merger events. These mergers are highly energetic $\left(10^{63}-10^{64} \mathrm{erg}\right)$ and provide significant energy input into the intra-cluster medium (ICM) through shocks and turbulence which can lead to amplification of magnetic fields and acceleration of relativistic particles, thus triggering synchrotron emission in the ICM. We present results from a low frequency VLA study of the merging cluster Abell 754. Optical and X-ray data reveal that this cluster has undergone a major merger event. The radio data reveal two clear regions of diffuse synchrotron emission, one concentrated in the cluster center and the other located on the edge of a bar-like structure found in the X-rays. We discuss the spectral index of the relativistic particles and compare the radio and X-ray emission of Abell 754. Comparing the spatial and spectral distribution of the relativistic particles to the X-ray temperature maps provides additional information on the nature of the complex merger within this cluster.
\end{abstract}

\section{Cluster Soft Excess Emission in the XMM/Newton Era Richard Lieu (Alabama), Jonathan Mittaz (Alabama)}

\begin{abstract}
In the XMM Newton era, research on the topic of cluster soft excess takes a sharp upward turn. The phenomenon is clearly confirmed for many clusters, moreover its thermal warm gas origin is established for the outer parts of five clusters through the detection of O VII emission at the cluster redshift. Since this line is not present in the inner soft excess spectra, the warm gas may reside in filaments projecting from a cluster and accounting in total for a lot of 'missing baryons'. Nonetheless, the mystery of the inner soft excess, which is bright enough to remain clearly visible even in the cooling flow regions where the hot intra-cluster medium is very luminous, can only be explained as inverse Compton emission. By using the clusters A1795 and AS1101 as test cases, we modeled the central soft excesses with a power law and in this way evaluated the observed cosmic rays pressure. In each case the parameter values indicate that the pressure is at least sufficient for equipartition between cosmic
\end{abstract}


rays and gas. Thus there is compelling evidence that the soft excess emission in clusters centers and the cooling flow riddle are really one and the same problem.

\title{
Deep WSRT 0.35-1.4 GHz Imaging of the Perseus Cluster Ger de Bruyn (ASTRON) \& Michiel A. Brentjens (Gröningen)
}

\begin{abstract}
We present new radio observations at $0.35 \mathrm{GHz}$ of the Perseus cluster taken in Dec 2002 with the new wide-band correlator at the WSRT. The data have been combined with existing, extremely high dynamic range observations at $1.4 \mathrm{GHz}$, to analyze the diffuse synchrotron emission from the existing radio galaxy population, the halo from NGC1275 and its cooling flow and the intra-cluster medium extending to $1 \mathrm{Mpc}$ radius. The relation between the radio and X-ray morphologies can be compared in detail. We have also detected widespread polarized emission which we interpret as due to a combination of Galactic foreground emission and Thomson scattered emission from the central radio source off the hot cluster gas. Our wide-band data furthermore allow us to study the radial dependence of the cluster and foreground Rotation Measure.
\end{abstract}




\section{POSTERS*}

\section{CLUSTER FORMATION}

Clusters at $z>1$ observed with XMM and Chandra--Christophe Adami Spectroscopy of the ESO distant cluster survey (EDISCS) - Claire Halliday The ellipticity of galaxy clusters - Piotr Flin

The morphological decomposition of Abell 868 -Simon Driver

The ACS Virgo cluster survey - Michael West

The XMM LSS survey--initial spectroscopic results -Jon Willis

Galaxy overdensities in the Shapley Supercluster - Lin-wen Chen

Galaxy clusters and cosmology - Thomas Reiprich

MACS: the evolution of very massive clusters out to $z=0.7$ - Harald Ebeling

MACS J0717.5+3745: a merging massive cluster at $z=0.55-$ Elizabeth Barrett

Temperature structure and point sources in Abell 1650 --Koujun Yamashita

Merging clusters as unidentified 3EG sources - Wataru Kawasaki

Arc statistics in galaxy clusters - Masamune Oguri

The Asiago-ESO/RASS QSO survey - Alessandro Omizzolo

High $-z$ clusters traced by groups of radiosources - Garret Cotter

MHD simulations of the formation of cold fronts - Naoki Asai

\section{CLUSTER GALAXIES}

Origin of $\mathrm{E}+\mathrm{A}$ galaxies in clusters - Kenji Bekki

Stellar population gradients of early-type galaxies in Coma - Daniel Thomas

The local Butcher-Oemler effect - Roberto De Propris

Extremely red objects at $z<1.5-$ Marian Suran

Rings in radio galaxies: a multiwavelength approach --Nectaria Gizani

The kinematics of spirals in distant galaxy clusters -Jochen Heidt

A survey of nearby clusters with the INT - Michael Pracy

Dying radio galaxies in clusters - Paola Parma

Radio and FIR properties of nearby X-ray cluster galaxies - Naveen Reddy Large scale radio jets in the galaxy cluster Abell S0102 - Miroslav Filipovic Cen A and its interaction with the X-ray-emitting ISM - Diana Worrall Evolutionary synthesis for galaxy transformation in clusters - Uta Fritze-v. Alvensleben

\section{INTRACLUSTER MEDIUM}

Optical spectrocopy of the cooling flow cluster 2A 0335+096 - Richard Gelderman

XMM-Newton detection of the WHIM in clusters - Jelle Kaastra

Detailed radio and optical observations of A3667 - Melanie Johnston-Hollitt

Spectral index of the radio halos in A665 and A2163 - Luigina Feretti

Measuring cluster magnetic fields - Nectaria Gizani

Simulating magnetic fields in galaxy clusters - Klaus Dolag

Simulations of clusters of galaxies with thermal conduction - Viktor Ziskin

Non-thermal hard X-ray excess in the Coma cluster - Roberto Fusco-Femiano

${ }^{*}$ Displayed posters only; to save space, only first authors are given here 